\title{
SATISFACCIÓN Y MOTIVACIÓN EN DESTINOS CULTURALES: TIPOLOGÍA DE LOS TURISTAS ATRAÍDOS POR EL PATRIMONIO INMATERIAL EN CUENCA (ECUADOR)*
}

\author{
SATISFACTION AND MOTIVATION IN CULTURAL DESTINATIONS: \\ TOWARDS THOSE TOURISTS ATTRACTED BY INTANGIBLE HERITAGE \\ IN CUENCA (ECUADOR)
}

\author{
José Prada Trigo ${ }^{* *}$ y Sandra Pesántez Loyola***
}

\begin{abstract}
El patrimonio inmaterial, asociado de manera creciente a la oferta turística de un territorio, es hoy un elemento que atrae cada vez a más visitantes. Sin embargo, existen aún pocos trabajos que aborden cuestiones como la motivación de los turistas, especialmente en contextos como el latinoamericano. El objetivo de la presente investigación es analizar la relación existente entre la motivación y el grado de satisfacción obtenido por los turistas que visitaron los lugares relacionados con el sombrero de paja toquilla (reconocido como Patrimonio Inmaterial de la Humanidad por la Unesco en 2012) en la ciudad de Cuenca (Ecuador). El trabajo realiza una segmentación de los turistas a base de tres dimensiones: una cultural, otra relacionada con el ocio y la última en referencia a cuestiones sociolaborales. Los resultados, analizados mediante análisis factorial, análisis cluster y análisis de varianza (ANOVA), evidencian que la dimensión cultural de la motivación es la más importante, siendo, sin embargo, la motivación por cuestiones de ocio la que otorga una mejor valoración al conocimiento del sombrero de paja toquilla, la dimensión patrimonial de Cuenca o la satisfacción con el viaje.
\end{abstract}

Palabras claves: Turismo cultural inmaterial, motivación, satisfacción, sombrero de paja toquilla, Cuenca.

The intangible heritage increasingly associated with tourism in a territory is today, an element that is attracting more and more visitors. However, there are still few studies that address issues such as the motivation of these tourists, especially in contexts like those of Latin America. The aim of this research is to analyze the relationship between the motivation and satisfaction obtained by tourists who have visited the places associated with the Panama hat (recognized as an Intangible Heritage by UNESCO in 2012) in the city of Cuenca (Ecuador). The work creates a segmentation of tourists based on three dimensions: the cultural, another related to leisure and the last in reference to social and labor issues. The results, where a factorial analysis, a cluster analysis and an analysis of variance (ANOVA) were applied, show that the cultural aspect of motivation is the most important, being, however, the motivation for leisure issues which gives one a better assessment of their knowledge of the Panama hat, Cuenca's heritage or satisfaction with the trip.

Key words: Intangible cultural tourism, motivation, satisfaction, Panama hat, Cuenca.

\section{Introducción}

El turismo cultural relacionado con el patrimonio inmaterial se ha convertido en una interesante y creciente oportunidad de desarrollo para los territorios, transformando la identidad de una sociedad en un elemento generador de recursos económicos y que permite la promoción y difusión del conocimiento sobre el lugar al que estos se asocian (Herrero et al. 2004). En la actualidad, las festividades, tradiciones, gastronomía, estilos de vida y la producción local son elementos que transforman los destinos en sitios de interés turístico y su demanda es cada vez mayor, pues el turista busca experiencias distintas y auténticas en lugares nuevos y genuinos (Timothy y Boyd 2006), siendo la "autenticidad" un componente valorado a la hora de realizar una visita turística. En este sentido, según Richards (1996), el turismo cultural debe considerarse prioritario en las decisiones estratégicas de cada localidad debido a su crecimiento actual y futuro.

Esta relación entre patrimonio inmaterial y turismo ha sido estudiada en los últimos años debido a la importancia que ha cobrado recientemente y

\footnotetext{
* Resultado proyecto: Turismo y patrimonio inmaterial: El sombrero de paja toquilla.

** Universidad de Concepción, Facultad de Arquitectura, Urbanismo y Geografía. Concepción, Chile. Correo electrónico: jprada@udec.cl

*** Universidad de Cuenca, Facultad de Ciencias de la Hospitalidad. Cuenca, Ecuador. Correo electrónico: spesantezl@yahoo.com
} 
al incremento de diferentes manifestaciones de este tipo en la lista de la UNESCO. Dentro de los trabajos relativos a turismo cultural podemos encontrar estudios más enfocados hacia su definición conceptual (Poria, Reichel y Cohen 2013; Timothy y Boyd 2006; MacCannell 1976; Apostolakis 2003), o casos más específicos en los que se busca definir el perfil sociodemográfico del turista (Chen y Chen 2010; Correia et al. 2013; Antón et al. 2014; Nguyen y Cheung 2014). Dentro de este último grupo, el interés de estos trabajos se concibe como un mecanismo para mejorar la oferta turística de los destinos, siendo una forma de aproximarse a la realidad existente en torno al turismo cultural inmaterial. En estos trabajos, el perfil de los turistas que visitan destinos culturales se ha relacionado con personas de nivel educativo alto y con un nivel de ingreso igualmente elevado.

Sin embargo, cuestiones como las motivaciones que inciden en la elección de un determinado destino turístico y su nivel de satisfacción aún no cuentan con demasiados estudios. Existe muy poca información al respecto en este sentido, únicamente trabajos llevados a cabo en el ámbito europeo (Pérez-Gálvez et al. 2015). Por ello, el objetivo de este trabajo es precisamente identificar las motivaciones del turista que visita la ciudad de Cuenca $\mathrm{y}$, dentro de la misma, los elementos vinculados al sombrero de paja toquilla, inscrito como Patrimonio Inmaterial de la Humanidad en 2012, junto con el resultado de su visita por medio de la medición de los niveles de satisfacción. Este estudio, que se inscribe dentro del ámbito de la Geografía del Turismo, una de las ramas más dinámicas en el contexto de las investigaciones territoriales en la actualidad, se dirige hacia una ciudad intermedia dentro de la cordillera andina. Este espacio, pese a ser reconocido como Patrimonio Cultural de la Humanidad en 1999, es aún relativamente poco conocido por los turistas internacionales en comparación con Quito, quedando en ocasiones fuera de la visita tradicional a Ecuador, que comprende Quito-Otavalo-Guayaquil-Islas Galápagos. Por ello, el desarrollo de una oferta turística en torno al sombrero de paja toquilla puede resultar una oportunidad para reforzar el atractivo de Cuenca.

La metodología utilizada en esta investigación ha consistido en un estudio empírico por medio de la técnica del cuestionario. Mediante este trabajo de campo se pretende identificar la motivación para visitar los lugares vinculados al sombrero de paja toquilla, además de otros aspectos como la gastronomía, los sitios de interés y entretenimiento, etc. Para esto se utiliza un análisis factorial para la identificación de grupos homogéneos y, finalmente, medir el nivel de satisfacción obtenido por estos grupos. En virtud de esto, el artículo se estructura primeramente mediante una revisión de la literatura del patrimonio cultural inmaterial y su relación con el turismo, a continuación se realiza una introducción a la ciudad de Cuenca, su relación con el turismo y el sombrero de paja toquilla; y posteriormente, tras la presentación de la metodología, se realiza una interpretación estadística de los datos obtenidos, de forma que se combinan aspectos cualitativos y cuantitativos.

\section{Revisión de la literatura}

\section{Turismo y patrimonio inmaterial}

En los últimos años el concepto de patrimonio cultural inmaterial ha evolucionado en dos direcciones diferentes (Del Barrio et al. 2012). Por un lado ha aumentado la lista de lugares Patrimonio de la Humanidad, desde una inicial restricción a monumentos, edificios históricos o lugares arqueológicos hasta nuevas perspectivas centradas en la cultura, como jardines, formas de producción o entornos rurales. Por otro lado se han incorporado otros rangos de elementos inmateriales que pretenden reconocer diferentes identidades colectivas como costumbres, folklore o tradiciones orales y que también forma parte de la herencia cultural de los territorios (Kirshenblatt-Gimblett 2004).

El patrimonio cultural intangible o inmaterial ha sido descrito como las prácticas, representaciones, expresiones, conocimiento, artes, etc., que las comunidades, grupos e individuos reconocen como parte de su patrimonio cultural (Mohd et al. 2013). Este se transmitiría de generación en generación, siendo constantemente reelaborado por los grupos y comunidades como respuesta a sus entornos, a su interacción con la naturaleza y a su historia, proveyendo de un sentido de identidad y de continuidad (Ahmad 2006). Se corresponde, por tanto, con la cultura que la gente practica como parte de su vida cotidiana, creencias y perspectivas, actuaciones efímeras y eventos que no son objetos tangibles de la cultura (Kurin 2004). Las tradiciones basadas en realizaciones de una comunidad cultural y reconocidas como reflejo de las expectativas de la 
misma, es decir, de su identidad social y cultural y sus estándares de vida se consideran parte de este patrimonio cultural intangible (KirshenblattGimblett 2004). Este concepto se está desarrollando actualmente en relación con cuestiones vinculadas a la valoración cultural del territorio y el crecimiento del turismo (Ciarcia 2006; Timothy y Boyd 2006).

Otro aspecto importante del patrimonio cultural inmaterial es su conexión con la identidad de sus creadores y transmisores. De esta forma, este patrimonio no existe como entidad aislada, sino que su significatividad depende de estos actores y de su propensión a transmitir el conocimiento y saber hacer adquirido a generaciones más jóvenes (Giudici et al. 2013). En este sentido, varios trabajos señalan que la percepción de un patrimonio como auténtico es un factor crucial a la hora de la evaluación y percepción que este recibiría de los visitantes (Naoi 2004; Kolar y Zabkar 2007; Giudici et al. 2013). En este contexto, desde los años noventa del siglo XX la cultura se ha considerado un recurso para promocionar el turismo e incluso crear un clima propicio a las inversiones en relación con este sector. Desde esta perspectiva, la cultura ha dejado de ser un fin en sí misma y ha pasado a significar la promoción económica local, y el turismo se considera hoy un incentivo para la conservación de monumentos y la rehabilitación de los centros históricos (Steinberg 1996), atrayendo el interés de gente de otros lugares (Romero Cevallos 2005).

El turismo cultural se entiende como aquel tipo de turismo en el que el patrimonio cultural, tanto el pasado como el presente, están en el centro de la visita, sean manifestaciones tangibles o intangibles de la cultura (Richards 1996). Este turismo vendría inspirado fundamentalmente por tres causas: la valoración creciente de la cultura y el patrimonio histórico por parte de la sociedad; la tendencia a abandonar el modelo tradicional de "sol y playa" crecientemente masificado; y el proceso de tercerización que ha experimentado la economía, y que ha otorgado mayor importancia a los momentos dedicados al ocio (Sanz et al. 2001).

En ocasiones, la designación de un lugar como World Heritage Site (WHS) es percibido como una marca (Timothy 2011) o como una etiqueta (Yang et al. 2010), reconocimiento que tiene una notable atracción para el turista y, en numerosas ocasiones, significa una recomendación global para visitar dicho destino (Poria, et al. 2013), ya que los turistas viajan buscando experiencias auténticas y lugares genuinos
(Timothy y Boyd 2006), siendo necesario conocer la relación entre turista, motivación y destino. Entre los trabajos que analizan esta relación sobresalen los de Timothy y Boyd (2003), Di Giovine (2009), Timothy (2011) y Park (2014). La razón por la que se está incrementado de forma significativa la literatura científica en este campo es la importancia que ha adquirido este tipo de turismo (Poria et al. 2013), que ha llevado a una proliferación de estudios que buscan profundizar en dicha temática.

\section{Perfil del turista, motivación y percepción del destino}

Entre los objetivos de los trabajos que han intentado abordar el perfil de los turistas en destinos culturales se encuentra la definición de su perfil sociodemográfico (Chen y Chen 2010; Correia et al. 2013; Antón et al. 2014, Nguyen y Cheung 2014), habiéndose determinado que los turistas en destinos culturales tienen un nivel educativo elevado, en general con estudios universitarios, y que su renta es media o media-alta. Otro objetivo con un importante recorrido ha sido el de comprender las diferentes tipologías de los turistas patrimoniales, sus motivaciones, sus comportamientos, sus percepciones y sus experiencias, que son aspectos básicos para realizar una mejor gestión de los destinos y definir las correspondientes estrategias.

Desde esta perspectiva, se ha buscado establecer diferencias basadas en la valoración que estos turistas realizan de los servicios turísticos, estableciendo categorías fundamentadas principalmente en el origen de los turistas (Schoefield y Thompson 2007), pero también en la edad, género o nivel de estudios (McCleary et al. 2008), siendo los resultados desiguales y apuntando, en general, a una mayor influencia del origen como elemento diferenciador (Andereck y Caldwell 1994, Kee et al. 2007; McCleary et al. 2008). Sin embargo, existen pocos trabajos que incidan en la identificación de tipologías a base de la motivación del desplazamiento, habiéndose realizado algunos estudios en el caso del turismo gastronómico (Pérez-Gálvez et al. 2015) o del turismo en resorts naturales (Meng et al. 2006).

De esta forma, McKercher (2002) señala que las tipologías de turistas culturales pueden plantearse en relación con dos criterios: la motivación del viaje o razón del mismo y el nivel de las experiencias vividas en el destino. Desde esta perspectiva, existen algunos trabajos que han explorado este tema, 
generando distintas categorías según la importancia de estos elementos en cada turista en función de las motivaciones para visitar un lugar o de las razones para desplazarse hasta ese sitio (Sildelberg, 1995; Jansen-Verbeke, 1997; Nyaupane y Andereck 2014). Incluso con estos precedentes los trabajos vinculados a las motivaciones para la visita de sitios relacionados con el patrimonio cultural inmaterial son aún un campo por explorar.

Junto con las motivaciones de los turistas, es necesario a su vez analizar los atributos del destino, ya que el conjunto que conforma la totalidad de los visitantes puede encontrarse motivado por diferentes razones en su desplazamiento hacia un mismo lugar (Breakey 2012). En este sentido, según Abuamoud et al. (2014), la demanda de lugares caracterizados por su importancia de herencia patrimonial está también muy influenciada por los servicios prestados por las autoridades y por la disponibilidad y viabilidad de la comunidad local por estimular el turismo en estas zonas. Siguiendo a Vong y Ung (2012), existen cuatro factores relacionados con el desarrollo del turismo en estos lugares: la historia y la cultura del lugar, las facilidades que encuentren los turistas tanto para llegar al destino como ya en el propio lugar, la interpretación del patrimonio, y los atractivos que esta herencia patrimonial suponga.

En este sentido, en determinados sitios los turistas encuentran que la herencia patrimonial no forma parte de su propia cultura, convirtiéndose en mero observador de un patrimonio excepcional y universal (Saipradist y Staiff 2007), lo que implica que es necesario, en estas situaciones, considerar los diferentes contextos culturales para encontrar vías de entendimiento entre el visitante y el patrimonio, y la herencia cultural del lugar (Saipradist y Staiff 2007). En definitiva, es necesario reforzar la comprensión del WHS para las diferentes culturas (Poria et al. 2013), sobre todo cuando se trata de lugares visitados por turistas internacionales (Tucker y Carnegie 2014).

Para entender el comportamiento de los turistas en un espacio es necesario conocer el vínculo entre ellos y este último. Hoy se considera que el énfasis en los estudios acerca del turismo cultural debe ponerse en la naturaleza personal, subjetiva y sensorial que tiene el turismo cultural, donde los turistas se convierten en actores, dando forma a sus propias experiencias en entornos culturales y patrimoniales (Gelbman y Ron 2009). Según estos autores, significados personales e historias de vida influyen a la hora de entender este tipo de turismo, dando lugar a diferentes variantes como el turismo de legado o de herencia, que busca el significado personal de viajes emprendidos hacia destinos vinculados al pasado del turista (McCain y Ray 2003), o el turismo espiritual, donde el significado preciso del viaje depende de las propias creencias del turista (Norman 2011). En cualquier caso, resulta evidente que la tipología del turista cultural aparece tan compleja como las propias tipologías de turismo cultural y como las múltiples motivaciones para visitar un destino.

En relación con estos aspectos, el auge del turismo cultural se puede deber en parte al aumento de visitantes culturales "ocasionales", que dedican parte de sus vacaciones a visitar lugares culturales, en contraste con los visitantes culturales "específicos" que viajan expresamente para consumir cultura, pero eso no quiere decir que la gente se interese más por la cultura, siendo la "edudistracción" una de las motivaciones de gran parte de los que visitan las atracciones culturales (Richards 2001).

\section{Satisfacción del turista}

La satisfacción del turista es uno de los principales tópicos en el campo de las investigaciones en turismo, dependiendo, en gran medida, de los atributos del propio destino como podrían ser las facilidades del mismo, el conocimiento y su novedad (Correia et al. 2013). A su vez, la satisfacción está relacionada con las expectativas previas de los turistas respecto de su viaje (Antón et al. 2014). En este sentido, la satisfacción en este tipo de destinos está determinada por la experiencia global que incluye aspectos relacionados con cuatro factores: ocio, cultura, educación e interacción social (Chen y Chen 2010). Existe, además, una relación directa entre la satisfacción con el viaje y el deseo de volver al mismo, es decir, la lealtad al destino, entendiéndola como la intención de repetir el viaje y recomendar el mismo (Antón et al. 2014).

La variable clave de la lealtad hacia un destino turístico es la satisfacción del turista, tanto como elemento que dirige directamente la intención de regresar, como en las recomendaciones a terceras personas, o en su papel de variable modeladora de la relación entre imagen que se tiene de un destino y su lealtad al mismo. De esta forma, una experiencia positiva en cuanto a servicio, productos turísticos, viaje y otros recursos que un destino 
turístico puede proporcionar, generaría una mayor intención del turista en repetir la visita, además de una buena promoción del destino (Antón et al. 2014). En este sentido, la satisfacción y la intención de volver a un destino están en parte determinados por la valoración de las características del destino (Alegre y Garau 2010), siendo elementos básicos para evaluar el desempeño de un lugar o destino turístico (Yoon y Uysal 2005).

Según diferentes estudios, la satisfacción dependería, de esta forma, tanto de las expectativas hacia un destino como de la valoración del destino en sí (Huh et al. 2006; Yoon y Uysal 2005). Otros autores señalan el concepto de "valor percibido" (perceived value) a la hora de referirse a la evaluación realizada por un cliente a partir de la comparación entre las ventajas que puede obtener de un producto o servicio (en este caso de la visita a un destino turístico) y los costes o sacrificios (económicos y no económicos) que conlleva esta (Forgas-Coll et al. 2012). Joppe et al. (2001) señalan que la búsqueda de una mayor calidad en el sector turístico requiere el desarrollo de formas más específicas para medir la calidad de los servicios, existiendo importantes diferencias en la ratio de satisfacción según el origen de los visitantes, aunque podrían añadirse otros atributos como la motivación de estos. Desde esta perspectiva, es fundamental determinar las diferentes motivaciones para visitar un lugar vinculado al patrimonio cultural inmaterial en el espacio andino, como es el caso de estudio de Cuenca, analizado a continuación en su vinculación con el sombrero de paja toquilla.

\section{Descripción del área geográfica}

La ciudad de Cuenca, fundada como Santa Ana de los Cuatro Ríos de Cuenca, es una ciudad del centro austral de la República del Ecuador y es la capital de la provincia del Azuay (Figura 1). Se encuentra enclavada en un valle del sistema andino, a 2.550 metros sobre el nivel del mar, y rodeada de un sistema montañoso que alberga un extenso sistema lacustre que baña a la ciudad por medio de sus cuatro ríos, de donde proviene su nombre. Su población es de 331.000 habitantes, superando su área metropolitana el medio millón (INEC 2010).

Este espacio tiene una importancia histórica y cultural excepcional pues en ella se recogen fragmentos de las culturas que habitaron sus parajes, como la cañari a la que se sobrevino la expansión inca y luego la española (Vanegas 2008) y que dejaron atrás vestigios de arte, ciencia y religión. Conocida como la "Atenas del Ecuador" por sus innumerables poetas y destacados personajes del ámbito político, es sede de la Bienal de arte contemporáneo a nivel latinoamericano (Poloni-Simard 2006) y se ha desarrollado como un destino turístico cultural de gran importancia para el país en los últimos años, pese a su relativo aislamiento de las principales ciudades de Ecuador, Quito y Guayaquil.

Su planificación urbana en damero, que data del Renacimiento, el paisaje urbano, la fusión de sociedades y culturas y su excelente conservación como ciudad colonial le permitieron acceder a la honorífica declaratoria de Patrimonio Cultural de la Humanidad por la Unesco en 1999 (UNESCO 2015); siendo la segunda ciudad de Ecuador en ostentar este título después de la capital, Quito. Este reconocimiento y sus innumerables tradiciones han impulsado a la ciudad como destino turístico cultural del Ecuador, que recibe a diario visitantes motivados por su arquitectura de época republicana, sus fiestas tradicionales religiosas y laicas, los festivales internacionales de arte, sus numerosas exposiciones de cine y teatro, sus expresiones culturales resumidas en productos de alfarería, orfebrería, tejidos de distintos materiales como el mimbre y, sin lugar a dudas, el sombrero de paja toquilla ${ }^{1}$.

El sombrero de paja toquilla, uno de los íconos de la producción artesanal en el Ecuador es, sin duda, un elemento importante en el desarrollo de la ciudad de Cuenca y su región. Su elaboración nace en la zona costera del Ecuador, provincia de Manabí, en el 3.500 a.C. y luego fue adquirido por los indígenas de las provincias del Azuay y Cañar, principalmente, a partir de 1835 (MINTUR 2014). Su fino tejido y delicado acabado pronto lo catalogaron como un elemento apetecido y de gran renombre a nivel internacional convirtiéndose en un producto de exportación y ocupando el primer lugar de los productos exportados por el Ecuador en 1944, con el nombre de Sombrero de Panamá (MINTUR 2014).

El 5 de diciembre de 2012 el sombrero de paja toquilla fue declarado como Patrimonio Cultural Inmaterial de la Humanidad convirtiéndose en una gran oportunidad para el desarrollo del turismo local (Figura 2). Su proceso de fabricación así como la gran variedad de modelos y la extensa gama de colores atraen diariamente a turistas nacionales y extranjeros motivados por conocer sus orígenes, a 


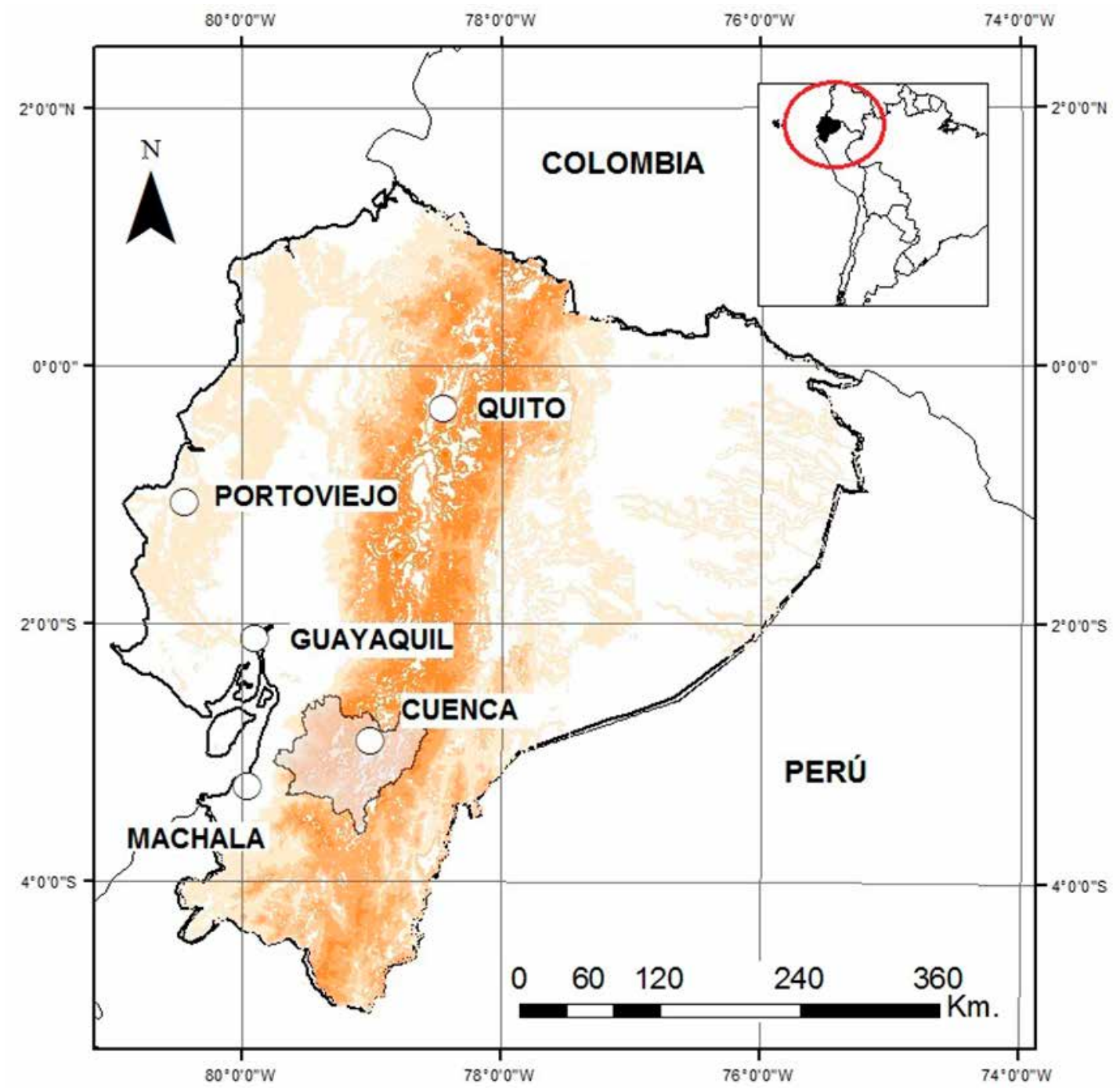

Figura 1. Localización de la ciudad de Cuenca, Ecuador. Fuente: Elaboración propia.

los maestros artesanos que elaboran diariamente estos preciados artículos y las empresas que han optado por desarrollarse en este sector económico aunque, como se dijo en el marco teórico, es posible también que existan diferentes razones para visitar este elemento de la ciudad de Cuenca.

\section{Metodología}

A nivel metodológico, se ha desarrollado un trabajo empírico con encuestas personales estructuradas, dirigidas a los turistas que se encontraban visitando la ciudad de Cuenca en diferentes puntos turísticos relacionados con el sombrero de paja toquilla. El cuestionario en papel ha sido la herramienta utilizada para la recogida de información. Este cuestionario, previamente a su implementación, pasó por un muestreo piloto y una mejora después de analizar los resultados. La población objetivo para este trabajo se ha identificado como los turistas ecuatorianos o extranjeros que se encontraban en la ciudad de Cuenca visitando lugares vinculados al sombrero de paja toquilla, durante octubre y noviembre de 2015. Como técnica de muestreo se ha utilizado el muestreo no probabilístico y de conveniencia. Los turistas encuestados han sido seleccionados tratando de abarcar distintas capas sociodemográficas y un perfil lo más amplio posible, pese a que el hecho 


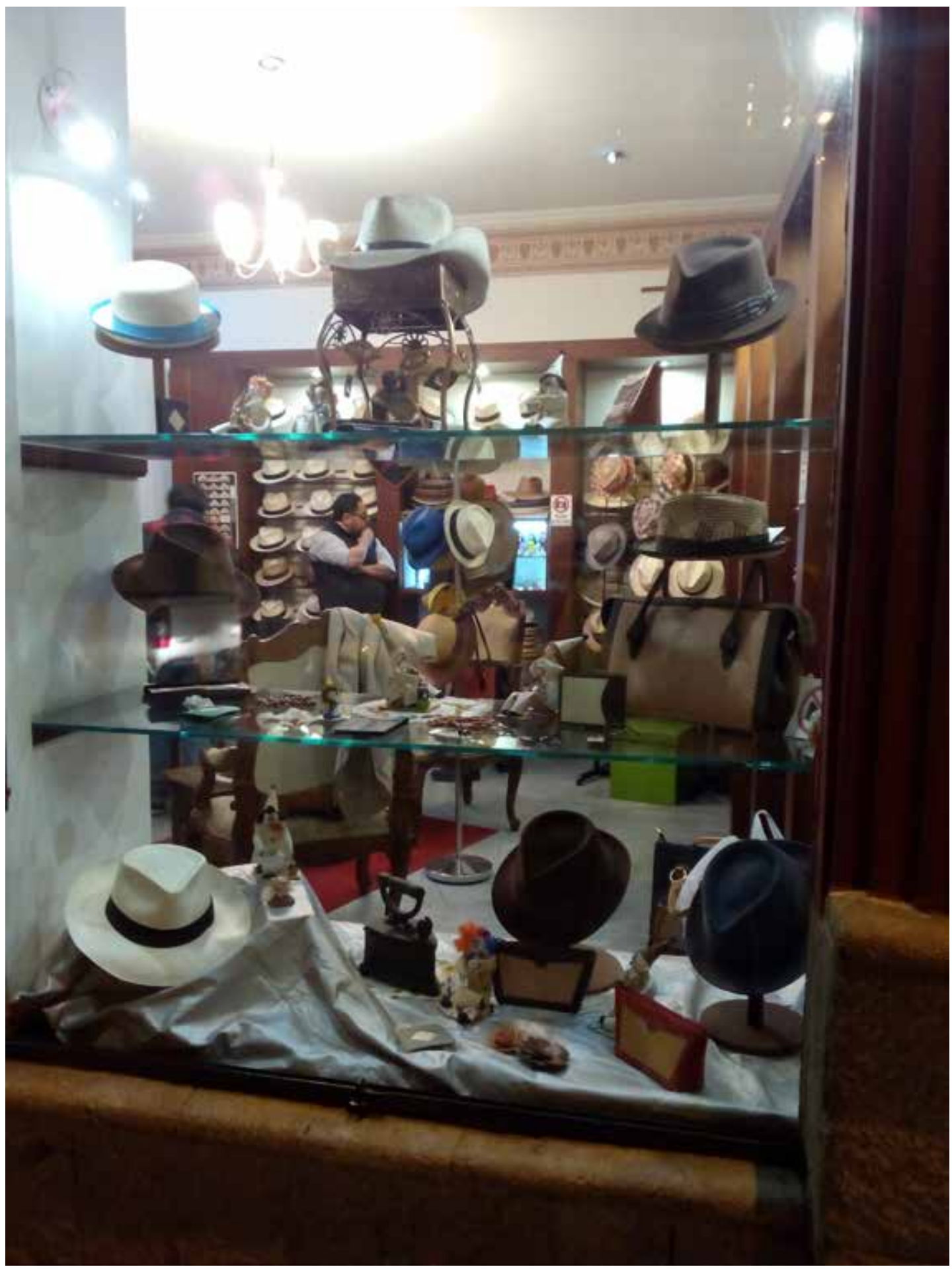

Figura 2. Tienda en el centro histórico de Cuenca donde se comercializa el sombrero de paja toquilla. Fuente: Elaboración propia. 
de realizar las encuestas mayoritariamente entre semana, ha supuesto un sesgo en favor de los turistas extranjeros. En la Tabla 1 aparecen los detalles de los aspectos técnicos de la investigación.

En cuanto al principal elemento analizado en este trabajo, la motivación para visitar los lugares vinculados al sombrero de paja toquilla de la ciudad de Cuenca se compuso de varias respuestas con una escala tipo Likert de 1 a 5, correspondiéndose 1 con "poco" y 5 con "mucho" en los motivos del viaje. Las diez preguntas realizadas respecto de esta cuestión tenían que ver con la visita a los lugares donde se fabrica el sombrero de paja toquilla, el aprendizaje de la fabricación del sombrero de paja toquilla, la degustación de la gastronomía de Cuenca, la visita a los museos de la ciudad, el entretenimiento, la relajación, el pasar un día fuera, el conocimiento de la ciudad de Cuenca, la visita a amigos o familiares y, por último, las cuestiones vinculadas con su trabajo o profesión. A continuación se aplicaron algunas preguntas, también con una escala tipo Likert de 5 puntos, acerca de la satisfacción respecto del sombrero de paja toquilla, la ciudad de Cuenca y el viaje en sí.

El análisis estadístico se ha centrado en las relaciones entre estas dos variables fundamentales en el proceso de decisión de la visita: la motivación y la satisfacción con la misma. Para ello se han utilizado principalmente el análisis factorial, el análisis de conglomerados y el análisis de la varianza (ANOVA con comparaciones múltiples post hoc). Para ello se ha recurrido al programa IBM SPSS Statistics 21. A continuación, se lleva a cabo el análisis e interpretación de los resultados principales.

La categorización de los encuestados según rango de edad, sexo, estudios, profesión, nivel de ingresos y origen (Tabla 2) mostró un perfil predominante de turistas con un nivel de educación alto $(75,7 \%)$, lo que confirma los datos de trabajos similares (Prada-Trigo et al. 2016), con un mayor número de hombres (7\% más) y con una tendencia al predominio de viajeros jóvenes (el 59,8\% de los viajeros tenía menos de 40 años). La mayoría de los mismos eran autoempleados y empleados en el sector privado, con una distribución muy homogénea entre los provenientes de Ecuador, América Latina, Europa y Norteamérica, lo que posiblemente también se relaciona con una distribución muy homogénea en los ingresos.

\section{Análisis de los resultados}

En este estudio se ha realizado un análisis factorial de la escala de motivación para identificar un pequeño número de factores explicativos de la motivación para visitar los lugares vinculados al sombrero de paja toquilla. En los resultados, que se presentan en la Tabla 3, se observa la existencia de tres diferentes grupos de motivaciones con un nivel de confianza del $95 \%$. Pese a que el interés principal se encuentra en las puntuaciones factoriales que se derivan de dichos componentes como herramienta para establecer la fuerza de las motivaciones de cada visitante, resulta de interés caracterizar cada unos de los factores localizados. El primer factor se ha denominado "motivos culturales" y está relacionado con el turista que plantea la visita como una forma de conocer la tradición del sombrero de paja toquilla, visitar los museos de la ciudad o degustar su gastronomía, siendo un turista muy cercano al concepto de "turista cultural" (Richards 2001; Silberberg 1995). El coeficiente alpha de Cronbach $(0,768)$ para los diferentes ítems que integran esta dimensión de motivación revela que representa una

Tabla 1. Diseño de la muestra de la investigación.

\begin{tabular}{ll}
\hline \multicolumn{1}{c}{ Universo } & \multicolumn{1}{c}{ Turistas y excursionistas en la ciudad de Cuenca } \\
\hline Ámbito & Municipal, Cuenca (Ecuador) \\
Método de recogida de información & Encuesta personal con cuestionario estructurado \\
Unidad muestral & Turistas y excursionistas de la ciudad de Cuenca \\
Tamaño de la población & No definido \\
Tipo de muestreo & No probabilístico de conveniencia \\
Nivel de confianza & $95 \%$ \\
Error muestral & $+/-3,4 \%$ \\
Trabajo de campo & Octubre y noviembre de 2015 \\
Número de encuestas válidas realizadas & 936 \\
\hline
\end{tabular}

Fuente: Elaboración propia. 
Tabla 2. Características sociodemográficas de la muestra.

\begin{tabular}{|c|c|c|}
\hline Variable & Ítem & $\%$ \\
\hline $\operatorname{Sexo}(N=910)$ & $\begin{array}{l}\text { Hombre } \\
\text { Mujer }\end{array}$ & $\begin{array}{l}53,5 \\
46,5\end{array}$ \\
\hline $\operatorname{Edad}(\mathrm{N}=922)$ & $\begin{array}{l}\text { Menor de } 30 \text { años } \\
30-39 \text { años } \\
40-49 \text { años } \\
50-59 \text { años } \\
60 \text { años o más }\end{array}$ & $\begin{array}{l}32,9 \\
26,9 \\
15,9 \\
11,0 \\
13,3\end{array}$ \\
\hline Nivel de estudios $(\mathrm{N}=901)$ & $\begin{array}{l}\text { Estudios básicos } \\
\text { Estudios medios } \\
\text { Estudios universitarios }\end{array}$ & $\begin{array}{c}2,4 \\
21,9 \\
75,7\end{array}$ \\
\hline Categoría profesional $(\mathrm{N}=910)$ & $\begin{array}{l}\text { Autoempleado } \\
\text { Empleado en el sector privado } \\
\text { Empleado en el sector público } \\
\text { Estudiante } \\
\text { Ama de casa } \\
\text { Jubilado } \\
\text { Otro }\end{array}$ & $\begin{array}{c}28,4 \\
24,0 \\
6,7 \\
15,7 \\
5,1 \\
13,3 \\
6,9\end{array}$ \\
\hline Ingresos mensuales $(\mathrm{N}=849)$ & $\begin{array}{l}\text { Alto }>2.500 \mathrm{USD} / \mathrm{mes} \\
\text { Medio-alto }>1.501<2.500 \mathrm{USD} / \mathrm{mes} \\
\text { Medio }>1.001<1.500 \mathrm{USD} / \mathrm{mes} \\
\text { Medio-bajo }>700<1.000 \mathrm{USD} / \mathrm{mes} \\
\text { Low }<700 \mathrm{USD} / \text { month }\end{array}$ & $\begin{array}{l}19,3 \\
19,6 \\
21,8 \\
19,9 \\
19,4\end{array}$ \\
\hline Origen $(\mathrm{N}=872)$ & $\begin{array}{l}\text { Ecuador } \\
\text { América Latina } \\
\text { Norteamérica } \\
\text { Europa } \\
\text { Otro }\end{array}$ & $\begin{array}{c}27,9 \\
22,5 \\
26,1 \\
21,4 \\
2,1\end{array}$ \\
\hline
\end{tabular}

Fuente: Elaboración propia.

subescala muy fiable. La importancia de este factor explica por sí solo el $31,82 \%$ de la varianza total de la matriz de motivaciones, es decir, casi un tercio de los turistas vendría atraído por estos motivos.

El segundo factor extraído, que se ha denominado "motivos de ocio", se relaciona con un turista que concibe la visita como un instrumento para entretenerse, relajarse, pasar un día fuera y escapar de la rutina, o conocer la ciudad. El coeficiente alpha de Cronbach $(0,708)$ para esta subescala también muestra una buena consistencia interna, explicando este factor por sí solo el 17,62\% de la varianza total de la matriz de motivaciones. Por último, se ha identificado un tercer factor, que se ha decido denominar "motivos sociolaborales", al vincularse a aquellas personas que conciben el viaje con el fin de visitar a la familia y amigos o por razones de trabajo, pero que aprovechan para conocer atractivos turísticos. El coeficiente alpha de Cronbach $(0,378)$ para los diferentes ítems que integran esta dimensión de motivación revela que representa una menor fiabilidad, lo que resulta lógico, al reunir dos elementos tan dispares como las visitas sociales y las cuestiones laborales, explicando este factor $12,24 \%$ de la varianza total de la matriz de motivaciones.

El estudio de las motivaciones proporciona fundamentos para establecer una segmentación del destino basado en la motivación que permita diferenciar entre grupos de visitantes. Para esto se ha recurrido a un análisis de conglomerados no jerárquico con las puntuaciones factoriales de los tres factores señalados arriba. Con el criterio de maximizar la varianza entre tipologías y minimizar la varianza dentro de cada tipología, la mejor solución es la que establece tres conglomerados (Tabla 4). La caracterización de los clusters a partir de las medias de las variables de motivación refleja que las medias comparadas no son iguales, pero no permite precisar dónde se encuentran las 
Tabla 3. Matriz de análisis factorial - Motivación de la visita.

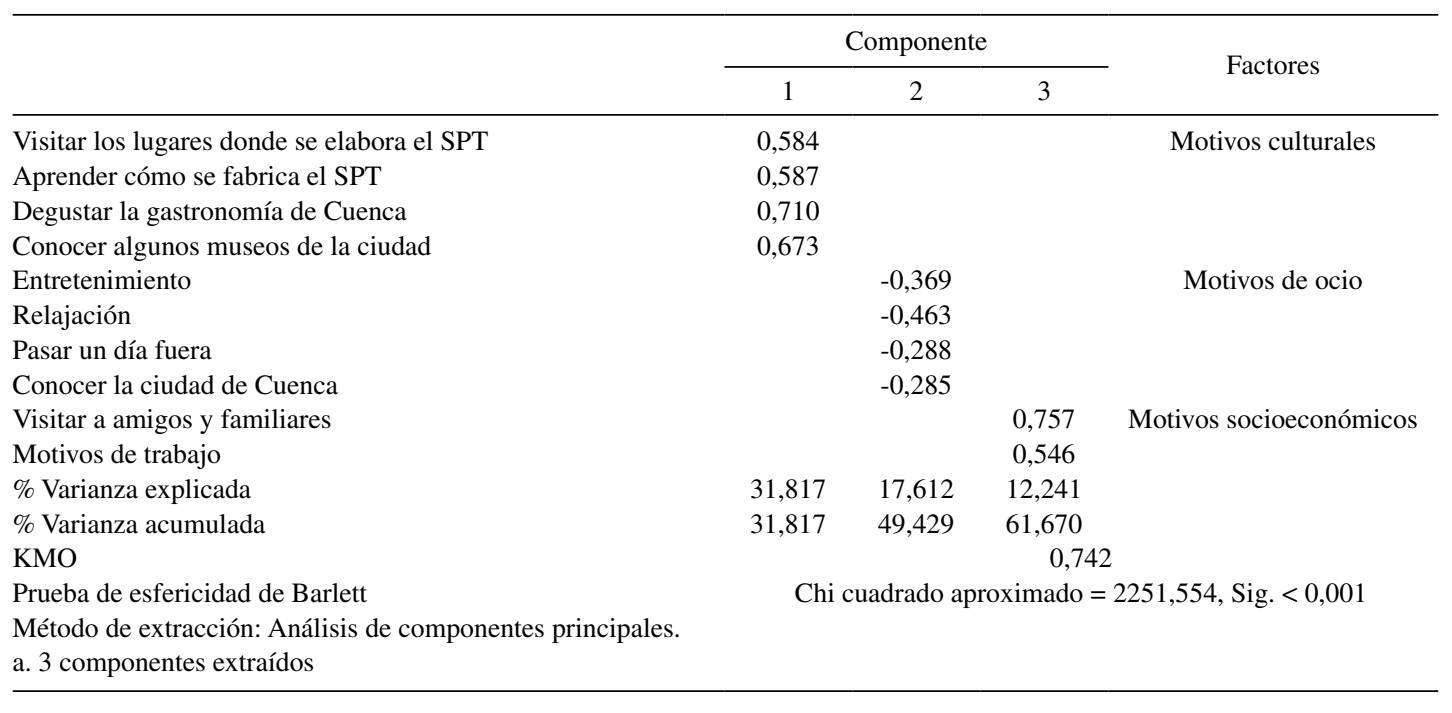

Fuente: Elaboración propia.

diferencias detectadas. La aplicación de una prueba ANOVA, que sirve para conocer si las diferencias en las puntuaciones medias resultan casuales o, por el contrario, son estadísticamente significativas, refleja una puntuación menor a 0,05 , por lo que los promedios de las variables motivacionales de los tres conglomerados comparados no serían iguales.

$\mathrm{Al}$ objeto de efectuar estas comparaciones no puede asumirse que las varianzas poblaciones son iguales, ya que el nivel crítico asociado al estadístico de Levene es menor que 0,05 para todos los casos, por lo que se rechaza la igualdad de varianzas. El estadístico F del ANOVA se sustenta en el cumplimiento de dos supuestos: homocedasticidad y normalidad. Basado en que no es posible asumir que las varianzas poblacionales sean iguales, se ha recurrido a los estadísticos de Brown-Forsythe y de Welch como alternativa al estadístico F del ANOVA (Tabla 5). Debido a que el nivel crítico asociado a ambos estadísticos en la Tabla 4 es menor que 0,05 , se puede rechazar la hipótesis de igualdad de medias y concluir que los promedios de las variables motivacionales de los tres conglomerados comparados no son iguales, por lo que existirían estos tres grupos claramente diferenciados.

El primero de los clusters presenta valores moderados en casi todas las variables motivacionales, anotando la mayor puntuación en los cuatro primeros ítems: visitar los lugares donde se elabora el sombrero de paja toquilla, aprender cómo se fabrica el sombrero de paja toquilla, degustar la gastronomía de Cuenca y conocer algunos museos de la ciudad, junto con conocer la ciudad de Cuenca, que es el más destacado. Por ello, este grupo podría identificarse con la tipología de turista cultural, que llega a la ciudad para conocerla, pero una vez allí se interesa por aspectos vinculados a la cultura en mayor medida que por el entretenimiento, la relajación, el visitar amigos o familiares o las cuestiones laborales. Este sería el cluster más reducido, con 167 turistas, existiendo una distancia entre conglomerados de 4,374 respecto al cluster 2 y de 3,524 con el tercer cluster.

El segundo cluster se relacionaría con valores más altos en los ítems vinculados a pasar un día fuera, el entretenimiento y la relajación, junto con otros aspectos también culturales como degustar la gastronomía de Cuenca o conocer algunos museos de la ciudad. Sin embargo, resulta también significativo el hecho de que visitar a amigos y familiares y motivos de trabajo también son relativamente altos, pudiendo interpretarse este grupo como el de turistas que visitan a familiares o conocidos o van a la ciudad por cuestiones laborales, pero una vez en Cuenca realizan algunas actividades turísticas variadas, que incluyen elementos culturales. La encuesta reflejó 300 turistas dentro de este grupo, los que mantenían una distancia de 3,805 con el 
Tabla 4. Caracterización cluster a partir de las medias de las variables de motivación.

\begin{tabular}{|c|c|c|c|c|c|c|c|}
\hline & \multicolumn{3}{|c|}{$\begin{array}{c}\text { Conglomerado } \\
\text { (puntuación media) }\end{array}$} & \multicolumn{2}{|c|}{ ANOVA } & \multicolumn{2}{|c|}{$\begin{array}{c}\text { Estadístico igualdad } \\
\text { de varianzas }\end{array}$} \\
\hline & 1 & 2 & 3 & $\Gamma$ & S. & I & S. \\
\hline & Media & Media & Media & & & & \\
\hline Visitar los lugares donde se elabora el SPT & 3,04 & 3,48 & 1,40 & 357,583 & $<0,001$ & 54,484 & 000 \\
\hline Aprender cómo se fabrica el SPT & 2,96 & 3,56 & 1,44 & 347,984 & $<0,001$ & 42,077 &, 000 \\
\hline Degustar la gastronomía de Cuenca & 3,31 & 4,56 & 3,41 & 126,562 & $<0,001$ & 51,771 &, 000 \\
\hline Conocer algunos museos de la ciudad & 3,26 & 4,28 & 3,18 & 87,881 & $<0,001$ & 25,823 &, 000 \\
\hline Entretenimiento & 2,47 & 4,39 & 3,68 & 172,633 & $<0,001$ & 20,046 &, 000 \\
\hline Relajación & 2,59 & 4,59 & 4,08 & 222,876 & $<0,001$ & 45,019 &, 000 \\
\hline Pasar un día fuera & 2,2 & 4,43 & 3,73 & 193,527 & $<0,001$ & 35,744 &, 000 \\
\hline Conocer la ciudad de Cuenca & 3,72 & 4,7 & 4,32 & 52,388 & $<0,001$ & 59,834 &, 000 \\
\hline Visitar a amigos y familiares & 1,83 & 3,34 & 2,20 & 61,077 & $<0,001$ & 27,143 &, 000 \\
\hline Motivos de trabajo & 2,29 & 2,02 & 1,37 & 29,839 & $<0,001$ & 92,406 &, 000 \\
\hline
\end{tabular}

Fuente: Elaboración propia.

Tabla 5. Pruebas robustas de igualdad de las medias variables de motivación.

\begin{tabular}{|c|c|c|c|c|c|}
\hline & & Estadístico $^{\mathrm{a}}$ & gl1 & $\mathrm{g} 12$ & Sig. \\
\hline \multirow{2}{*}{ Visitar los lugares donde se elabora el SPT } & Welch & 468,464 & 2 & 365,819 & , 000 \\
\hline & Brown-Forsythe & 306,998 & 2 & 417,611 &, 000 \\
\hline \multirow{2}{*}{ Aprender cómo se fabrica el SPT } & Welch & 434,351 & 2 & 374,515 & 000 \\
\hline & Brown-Forsythe & 308,657 & 2 & 456,081 & 000 \\
\hline \multirow{2}{*}{ Degustar la gastronomía de Cuenca } & Welch & 161,020 & 2 & 376,653 & 000 \\
\hline & Brown-Forsythe & 120,156 & 2 & 521,594 & 000 \\
\hline \multirow{2}{*}{ Conocer algunos museos de la ciudad } & Welch & 102,876 & 2 & 396,318 & ,000 \\
\hline & Brown-Forsythe & 81,839 & 2 & 521,911 & ,000 \\
\hline \multirow{2}{*}{ Entretenimiento } & Welch & 171,572 & 2 & 404,603 & 000 \\
\hline & Brown-Forsythe & 163,715 & 2 & 551,250 &, 000 \\
\hline \multirow{2}{*}{ Relajación } & Welch & 179,821 & 2 & 381,330 &, 000 \\
\hline & Brown-Forsythe & 195,194 & 2 & 441,590 &, 000 \\
\hline \multirow{2}{*}{ Pasar un día fuera } & Welch & 199,938 & 2 & 401,551 & ,000 \\
\hline & Brown-Forsythe & 185,965 & 2 & 561,360 &, 000 \\
\hline \multirow{2}{*}{ Conocer la ciudad de Cuenca } & Welch & 48,040 & 2 & 368,248 &, 000 \\
\hline & Brown-Forsythe & 45,159 & 2 & 417,917 & 000 \\
\hline \multirow{2}{*}{ Visitar a amigos y familiares } & Welch & 63,278 & 2 & 464,310 &, 000 \\
\hline & Brown-Forsythe & 65,712 & 2 & 743,600 &, 000 \\
\hline \multirow{2}{*}{ Motivos de trabajo } & Welch & 33,653 & 2 & 383,196 &, 000 \\
\hline & Brown-Forsythe & 26,756 & 2 & 473,192 &, 000 \\
\hline
\end{tabular}

Fuente: Elaboración propia.

tercer conglomerado y que puede asociarse a los turistas "culturales ocasionales" identificados en otros trabajos sobre la ciudad (Prada-Trigo et al. 2016).

Finalmente, el tercer cluster nos mostraría un tipo de turista centrado en conocer la ciudad de Cuenca, primando la relajación, el entretenimiento y pasar un día fuera; siendo característicos de un tipo de turista no cultural o, incluso, hedonista. El objetivo de este grupo es pasar un día relajado, disfrutando de algunos elementos característicos de la ciudad que pueden vincularse a la cultura -como es el caso de la gastronomía-, pero sin que esa sea su motivación principal. Este grupo, que presenta 303 
turistas en el caso de la encuesta realizada, muestra una distancia considerable respecto de los otros dos.

Finalmente, una vez establecidos los grupos de turistas, resulta necesario conocer su nivel de satisfacción. Para ello se identificaron tres preguntas relacionadas con la satisfacción respecto del conocimiento adquirido del sombrero de paja toquilla, con Cuenca como ciudad patrimonial y con el viaje en general. Los resultados (Tabla 6) reflejan, entre las tres preguntas, una menor satisfacción general con el sombrero de paja toquilla, seguida de una satisfacción mayor con el viaje, siendo la nota más alta la que hace referencia a la satisfacción con Cuenca como ciudad Patrimonio de la Humanidad. Por conglomerados, destaca el hecho de que la satisfacción con el sombrero paja toquilla sea más alta en los conglomerados 1 y 2 (especialmente en el segundo) que en el caso del conglomerado 3 , posiblemente porque sus intereses van en otra dirección. En el caso de la satisfacción con Cuenca como ciudad patrimonial, por el contrario, la satisfacción más baja la presenta precisamente el turista cultural, o conglomerado 1, siendo superior en el caso del segundo conglomerado, lo que nos puede indicar un mayor disfrute por parte de aquellos turistas que vienen por diversas razones y terminan sintiéndose muy satisfechos con la oferta cultural. Esta misma división se corresponde también con la satisfacción con el viaje, que indicaría una menor calificación por parte de los turistas culturales. Por último, las pruebas de robustez respecto de las medias de las tres variables de satisfacción muestran nuevamente que las puntuaciones en los promedios de estas en los tres conglomerados comparados son estadísticamente significativas (Tabla 7).

\section{Conclusiones}

La mayor competencia entre territorios ha llevado a buscar nuevas fuentes de desarrollo económico, tratando estos de diversificar su economía y de generar empleo en sectores distintos a los tradicionales. Entre estas opciones, el turismo cultural ha emergido en los últimos años como uno de los sectores de mayor crecimiento en cuanto a atención por parte de los turistas. Sin embargo, pese a este rápido desarrollo, aún existen pocos trabajos en determinados espacios (como América Latina) o acerca de ciertos temas (como las motivaciones) para visitar estos destinos asociados al patrimonio inmaterial.

En este sentido, en el presente artículo se han estudiado las principales motivaciones del turista para aproximarse hacia los lugares vinculados al sombrero de paja toquilla en la ciudad de Cuenca,

Tabla 6. Caracterización conglomerados a partir de las variables de satisfacción.

\begin{tabular}{|c|c|c|c|c|c|c|c|}
\hline & \multicolumn{3}{|c|}{ Conglomerado } & \multicolumn{2}{|c|}{ ANOVA } & \multicolumn{2}{|c|}{$\begin{array}{l}\text { Estadístico } \\
\text { igualdad de } \\
\text { varianzas }\end{array}$} \\
\hline & 1 & 2 & 3 & $\mathrm{~F}$ & Sig. & Levene & Sig. \\
\hline Satisfacción sombrero paja toquilla & 4,0252 & 4,2424 & 3,5341 & 36,3910 & 0,0000 & 18,741 & ,000 \\
\hline Satisfacción Cuenca como ciudad Patrimonio Humanidad & 4,4051 & 4,6121 & 4,4686 & 4,7453 & 0,0090 & 3,307 & 037 \\
\hline Satisfacción viaje & 4,3025 & 4,5946 & 4,3480 & 9,3944 & 0,0001 & 8,060 & , 000 \\
\hline
\end{tabular}

Fuente: Elaboración propia.

Tabla 7. Pruebas robustas de igualdad de las variables de satisfacción

\begin{tabular}{|c|c|c|c|c|c|}
\hline & & Estadístico $^{\mathrm{a}}$ & gl1 & $\mathrm{g} 12$ & Sig. \\
\hline \multirow[t]{2}{*}{ Satisfacción sombrero paja toquilla } & Welch & 34,494 & 2 & 397,071 &, 000 \\
\hline & Brown-Forsythe & 36,180 & 2 & 595,408 &, 000 \\
\hline \multirow[t]{2}{*}{ Satisfacción Cuenca como ciudad Patrimonio Humanidad } & Welch & 5,118 & 2 & 414,109 & ,006 \\
\hline & Brown-Forsythe & 4,869 & 2 & 641,729 & ,008 \\
\hline \multirow[t]{2}{*}{ Satisfacción viaje } & Welch & 11,128 & 2 & 430,286 &, 000 \\
\hline & Brown-Forsythe & 9,892 & 2 & 672,073 &, 000 \\
\hline
\end{tabular}

a. Distribuidos en F asintóticamente.

Fuente: Elaboración propia. 
que abarca la visita a tiendas, museos y otros sitios relacionados con este producto. La posibilidad de realizar esta actividad en una ciudad como Cuenca, de tamaño relativamente pequeño y con una marcada concentración de sus atractivos turísticos, permite presuponer que la motivación para ir hacia estos lugares puede ser variada, no siendo necesario desplazarse exprofeso a Cuenca para esta actividad, que puede integrarse dentro de un conjunto de razones más amplio. En este artículo se muestra la evidencia respecto de la diversidad de razones por las que los visitantes optan por incluir elementos culturales inmateriales en su visita a Cuenca.

Basado en ellas, se han identificado tres dimensiones motivacionales claras a la hora de visitar la ciudad y los sitios vinculados al sombrero de paja toquilla. Una primera, que se ha denominado "cultural" y que representa el 31,82\% de la muestra, respondiendo a un grupo de turistas altamente motivados por el conocimiento de la cultura y las tradiciones vinculadas a la ciudad de Cuenca (no solamente el sombrero de paja toquilla, sino también la gastronomía o los museos). El segundo de los segmentos, que se ha denominado "de ocio", representa el $17,62 \%$ de la muestra y se corresponde con un turista que viene más motivado por el hecho de pasar un día agradable, relajarse, entretenerse, pasar un día fuera, conocer la ciudad y, dentro de su plan, puede incluir la visita a algún museo o elemento cultural o la degustación de la gastronomía cuencana. Finalmente, se identificó un tercer segmento, denominado "sociolaboral", que se corresponde con el $12,24 \%$ de la muestra, y que caracteriza a un turista que llega a la ciudad por motivos sociales, como visitar a la familia o amigos, o bien por razones económicas, motivos de trabajo. Una vez en la ciudad el visitante dedica una parte de su tiempo a conocer Cuenca, visitar algún museo o comer en un restaurante típico.

Los resultados obtenidos, en cuanto al grado de satisfacción, es, por lo general, alto, aunque existen diferencias significativas entre los segmentos turísticos identificados, estando claramente vinculada a las motivaciones de la visita (Tabla 6). El mayor grado de satisfacción corresponde a los turistas que llegan por motivos vinculados al ocio. Su grado de satisfacción con el sombrero de paja toquilla, la ciudad de Cuenca como lugar patrimonial y la visita en sí es más alto que en los otros dos grupos, pudiendo responder esto al hecho de que el ocio tiene un peso superior a la cultura en su motivación, lo que hace valorar con más generosidad esto último. A continuación, las otras dos categorías reflejan una satisfacción similar respecto de la ciudad de Cuenca y el viaje en general, estando la diferencia más significativa en la mayor satisfacción del turista "cultural" con el sombrero de paja toquilla, lo que podría indicar la importancia de este bien dentro de la oferta patrimonial de la ciudad de Cuenca, en tanto satisface ampliamente los requerimientos de quienes llegan buscando conocer la cultura "auténtica u original" de un lugar, como se indicaba en el marco teórico. Sin embargo, su menor valoración indica la necesidad de un esfuerzo en su promoción y puesta en valor, resultando, en este sentido, este trabajo orientativo para las políticas públicas en turismo.

La explotación de la información de la encuesta refleja, pese a la complejidad de los análisis, interesantes diferencias entre unos colectivos y otros en función de la motivación que lleva a realizar el viaje. Sin embargo, resulta necesario ampliar este tipo de estudios, acercándose a valoraciones más concretas, a la comparación con otras ciudades de Ecuador o de la región o por otras técnicas complementarias que aporten mayor información acerca de estos grupos identificados.

\section{Agradecimientos}

Este trabajo fue realizado a partir del proyecto Turismo y patrimonio inmaterial: el sombrero de paja toquilla, financiado por la Facultad de Ciencias de la Hospitalidad de la Universidad de Cuenca (Ecuador). Los autores desean igualmente agradecer a los evaluadores por sus valiosas contribuciones para enriquecer el texto final. 


\section{Referencias Citadas}

Abuamoud, I. N.; Libbin, J.; Green, J. y Alrousan, R.

2014 Factors affecting the willingness of tourists to visit cultural heritage sistes in Jordan. Journal of Heritage Tourism, 9 (2): 148-165.

Ahmad, Y.

2006 The scope and definitions of heritage: From tangible to intangible. International journal of heritage studies, 12 (3): 292-300.

Alegre, J. y Garau, J.

2010 Tourist satisfaction and dissatisfaction. Annals of tourism research, 37 (1): 52-73.

Andereck, K.L. y Caldwell, L.L.

1994 Variable Selection in Tourism Market Segmentation Models. Journal of Travel Research, 33 (2): 40-46.

Antón, C.; Camarero, C. y Laguna-García, M.

2014 Towards a new approach of destination royalty drivers: satisfaction, visit intensity and tourist motivation. Current Issues in Tourism, 35: 1-23.

Apostolakis, A.

2003 The convergence process in heritage tourism. Annals of tourism research, 30 (4): 795-812.

Breakey, N.M.

2012 Study in of World Heritage visitors: The case of the remote Riversleigh Fossil Site. Visitor Studies, 15 (1): 82-97.

Chen, C. y Chen, F.

2010 Experience quality, perceived value, satisfaction and behavioural intentions for heritage tourists. Tourism Management, 31, 29-35.

Ciarcia, G.

2006 La perte durable. Etude sur la notion de "patrimoine immatériel" LAHIC/Mission Ethnologie (Ministere de la Culture). Mission Ethnologie (Ministere de la Culture): 76.

Correia, A.; Kozak, M. y Ferradeira, J.

2013 From tourist motivations to tourist satisfaction. International Journal of Culture, Tourism and Hospitality Research, 7 (4): 411-424.

Del Barrio, M.J.; Devesa, M. y Herrero, L.C.

2012 Evaluating intangible cultural heritage: the case of cultural festivals. City, Culture and Society, 3 (4): 235-244.

Di Giovine, M.A.

2009 The Heritage-scape: UNESCO, World Heritage, and Tourism, Lexington: Books.

Forgas-Coll, S.; Palau-Saumell, R.; Sánchez-García, J. y

Callarisa-Fiol, L. J.

2012 Urban destination loyalty drivers and cross-national moderator effects: The case of Barcelona. Tourism Management, 33(6): 1309-1320.

Gelbman, A. y Ron, A.S.

2009 Heritage and cultural tourism: The present and future of the past. Tourism Geographies, 11(1): 127-129.

Giudici, E.; Melis, C.; Dessì, S. y Pollnow, B. F.

2013 Is intangible cultural heritage able to promote sustainability in tourism? International Journal of Quality and Service Sciences, 5 (1): 101-114.

Herrero, L.C.; Sanz, J.A.; Bedate, A.; Devesa, M. y Barrio, M.J. 2004 Turismo Cultural e Impacto Económico de Salamanca 2002. Ciudad Europea de la Cultura. Civitas Ediciones, Madrid.
Huh, J.; Uysal, M. y McCleary, K.

2006 Cultural/Heritage Destinations: Tourist Satisfaction and Market Segmentation. Journal of Hospitality \& Leisure Marketing, 14 (3): 81-99.

Hui, T. K.; Wan, D. y Ho, A.

2007 Tourists' satisfaction, recommendation and revisiting Singapore. Tourism management, 28(4): 965-975.

INEC

2010 VII Censo de Población y Vivienda. Quito: INEC.

Jansen-Verbeke, M.

1997 Urban tourism. Managing resources and visitors. Tourism, development and growth: The Challenge of Sustainability. Routledge, Londres.

Joppe, M.; Martin, D. y Waalen, J.

2001 Toronto's Image As a Destination: A Comparative Importance-Satisfaction Analysis by Origin of Visitor. Journal of Travel Research, 39: 252-260.

Kirshenblatt-Gimblett, B.

2014 Intangible Heritage as Metacultural Production. Museum International, 66 (1-4): 163-174.

Kolar, T. y V. Zabkar.

2007 The meaning of tourists'authentic experiences for the marketing of cultural heritage sites. Economic and Business Review for Central and South-Eastern Europe, 9 (3): 235.

Kurin, R.

2004 Safeguarding Intangible Cultural Heritage in the 2003 UNESCO Convention: a critical appraisal. Museum international, 56 (1-2): 66-77.

MacCannell, D.

1976 The tourist: A new theory of the leisure class. Berkerly. Universitiy of California Press.

McCain, Gary y Ray, Nina M.

2003 Legacy Tourism: The Search for Personal Meaning in Heritage Travel. Tourism Management, 24(6): 713-717.

McCleary, K.; Weaver, P. y Hsuc, C.

2008 The Relationship Between International Leisure Travelers' Origin Country and Product Satisfaction, Value, Service Quality, and Intent to Return. Journal of Travel \& Tourism Marketing, 21 (2-3): 117-130.

McKercher, B.

2002 Towards a classification of cultural tourists. International journal of tourism research, 4(1): 29-38.

Meng, F.; Tepanon, Y. y Uysal, M.

2008 Measuring tourist satisfaction by attribute and motivation: The case of a nature-based resort. Journal of Vacation Marketing, 14(1): 41-56.

\section{MINTUR}

2014 El sombrero de paja toquilla, Patrimonio Cultural Inmaterial de la Humanidad. Quito, 8 agosto 2014. Documento electrónico disponible en: www.turismo.gob. ec/

Mohd, N.; Zaki, S.; Saniah, S. y Syed H.

2013 Between Tourism and Intangible Cultural Heritage. Procedia - Social and Behavioral Sciences, 85 (20): 411-420.

Naoi, T.

2004 Visitors' evaluation of a historical district: the roles of authenticity and manipulation. Tourism and Hospitality Research, 5(1): 45-63. 
Nguyen, T.H.H. y Cheung, C.

2014 The classification of heritage visitors: a case of Hue City, Vietnam. Journal of Heritage Tourism, 9 (1): 35-50. Norman, A.

2011 Spiritual tourism: Travel and religious practice in western society. Nueva York: Bloomsbury Publishing.

Nyaupane, G.P. y Andereck, K.L.

2014 Visitors to cultural heritage attractions: an activity-based integrated typology. Tourism Culture \& Communication, 14(1): 17-26.

Park, H.Y.

2014 Heritage tourism. Routledge, Londres.

Pérez-Gálvez, Muñoz-Fernández y López-Guzmán

2015 Motivación y satisfacción turística en los festivales del vino: XXXI ed. cata del vino Montilla-Moriles, España. Tourism \& Management Studies, 11(2): 7-13.

Poloni-Simard, J.

2006 El Mosaico Indígena, Quito, AbyaYala

Poria, Y.; Reichel, A. y Cohen, R.

2013 Tourists perceptions of World Heritage Site and its designation, Tourism Management, 35: 272-274.

Prada-Trigo, J.; Armijos, D.; Crespo, A. y Torres, L.

2016 Ciudades patrimoniales, turismo cultural y perfiles de los visitantes: algunas consideraciones a partir del caso de estudio de Cuenca (Ecuador), Lurralde: investigación y espacio, 39: 199-216.

Richards, G.

2001 Cultural Attractions and European tourism, Wallingford: CABI.

Richards, G. (Ed.).

1996 Cultural tourism in Europe. Wallingford: CABI.

Romero Cevallo, R.

2005 ¿Cultura y desarrollo? ¿Desarrollo y cultura? Propuestas para un debate abierto. Cuadernos PNUD, Serie de Desarrollo Humano, No. 9. Recuperado de http://unesdoc.unesco.org/ images/0014/001440/144076s.pdf

Saipradist, A. y Staiff, R.

2007 Crossing the cultural divide: Western visitors and Interpretation at Ayutthaya World Heritage Site, Thailand. Journal of Heritage Tourism, 2 (3): 211-224.

Sanz, J.A.; Herrero, L.C. y Bedate, A.M.

2001 Turismo cultural y Patrimonio Histórico aplicación multivariante al estudio de la demanda. Estudios turísticos. 150(1): 113-132.
Schofield, P. y Thompson, K.

2007 Visitor motivation, satisfaction and behavioural intention: the 2005 Naadam Festival, Ulaanbaatar. International Journal of Tourism Research, 9: 329-344.

Silberberg, T.

1995 Cultural tourism and business opportunities for museums and heritage sites. Tourism management, 16(5): 361-365.

Steinberg, F.

1996 Conservation and rehabilitation of urban heritage in developing countries. Habitat International, 20(3): 463-475.

Timothy, D.J.

2011 Cultural heritage and tourism. Channel View Publications, Londres.

Timothy, D.J. y Boyd, S.W.

2006 Heritage tourism in the $21^{\text {st }}$ Century: valued traditions and new perspectives, Journal of Heritage Tourism 1 (1): $1-16$.

Tucker, H. y Carnegie, E.

2014 World heritage and the contradictions of 'universal value'. Annals of Tourism Research, 47: 63-76.

UNESCO

2005 Convention on the Protection and Promotion of the Diversity of Cultural Expressions 2005. París, 20 Octubre de 2005.

Vanegas R., Alejandro

2008 Cuenca en su raíz profunda, Coloquio, Revista Universidad del Azuay, 38: 7-12.

Vong, L.T.N. y Ung, A.

2012. Exploring critical factors of Macau's Heritage tourism: what heritage tourists are looking for when visiting the city's iconic heritage site. Asia Pacific Journal of Tourism Research, 17 (3): 231-245.

WTO-OMT.

1993 Recommendations and Tourism Statistics. WTO, Madrid. Yang, C.H.; Lin, H.L. y Han, C.C.

2010 Analysis of international tourist arrivals in China: The role of World Heritage Sites. Tourism management, 31 (6): 827-837.

Yoon, Y. y Uysal, M.

2005 An examination of the effects of motivation and satisfaction on destination loyalty: a structural model. Tourism Management, 26 (1): 45-56.

1 Los atractivos turísticos de Cuenca pueden consultarse en la web de la Fundación Municipal de Turismo: http:// cuencaecuador.com.ec/ 
